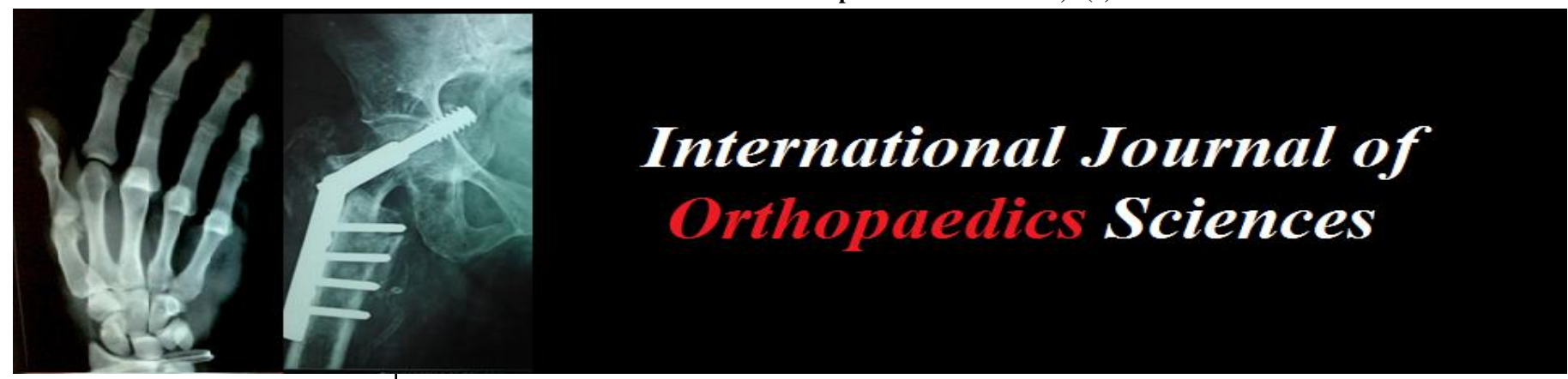

E-ISSN: 2395-1958

P-ISSN: 2706-6630

IJOS 2021; 7(1): 848-852

(C) 2021 IJOS

www.orthopaper.com

Received: 22-10-2020

Accepted: 24-12-2020

\section{Senthuren Isaac}

(1) Department of Orthopaedic Surgery, Sir Charles Gairdner

Hospital, Hospital Avenue, Nedlands,

WA 6009 , Australia

(2) Hollywood Private Hospital,

Monash Avenue, Nedlands, WA 6009

Australia

(3) The Joint Studio, Hollywood

Medical Centre, 85 Monash Avenue,

Nedlands WA, 6009, Australia

\section{Riaz JK Khan}

(1) Hollywood Private Hospital,

Monash Avenue, Nedlands, WA 6009

Australia

(2) The Joint Studio, Hollywood

Medical Centre, 85 Monash Avenue,

Nedlands WA, 6009, Australia

(3) Department of Medicine, Notre

Dame University, Fremantle, 32

Mouat Street, Fremantle WA, 6160

\section{Daniel P Fick}

(1) Hollywood Private Hospital,

Monash Avenue, Nedlands, WA 6009

Australia

(2) The Joint Studio, Hollywood

Medical Centre, 85 Monash Avenue,

Nedlands WA, 6009, Australia

Samantha Haebich

(1) Department of Orthopaedic

Surgery, Sir Charles Gairdner

Hospital, Hospital Avenue, Nedlands,

WA 6009, Australia

(2) The Joint Studio, Hollywood

Medical Centre, 85 Monash Avenue,

Nedlands WA, 6009, Australia

Michael Finsterwald

The Joint Studio, Hollywood Medical Centre, 85 Monash Avenue, Nedlands

WA, 6009, Australia

William Blakeney

The Joint Studio, Hollywood Medica Centre, 85 Monash Avenue, Nedland

WA, 6009, Australia

\section{Corresponding Author:}

Senthuren Isaac

(1) Department of Orthopaedic

Surgery, Sir Charles Gairdner

Hospital, Hospital Avenue, Nedlands,

WA 6009, Australia

(2) Hollywood Private Hospital,

Monash Avenue, Nedlands, WA 6009

Australia

(3) The Joint Studio, Hollywood

Medical Centre, 85 Monash Avenue,

Nedlands WA, 6009, Australia

\section{Computer navigation does not improve 10-year functional outcomes following total knee arthroplasty: A randomised clinical trial}

\section{Senthuren Isaac, Riaz JK Khan, Daniel P Fick, Samantha Haebich, Michael Finsterwald and William Blakeney}

DOI: $\underline{\text { https://doi.org/10.22271/ortho.2021.v7.i1m.2580 }}$

\section{Abstract}

Purpose: The use of computer-assisted surgery (CAS) in total knee arthroplasty (TKA) has been widely shown to be beneficial in improving prostheses alignment. The evidence of correlation to superior functional result, however is both sparse and conflicting.

Methods: TKA participants were randomised into one of three trial arms to compare implantation techniques; CAS, intramedullary instrumentation guides for femur and tibia or intramedullary guides for femur and extramedullary tibial jigs. This was a single centre trial, with one senior surgeon performing all surgeries through a standardised medial parapatellar approach and cemented prostheses. Participants were blinded to surgical technique, and post-operatively followed up for 10 years. Outcome measures included a Numerical Knee Pain Rating, Oxford Knee Score and Short Form Health Survey and Likert satisfaction score.

Results: 107 patients were successfully randomised for age, body mass index and knee function. Mean surgical times were 112 minutes for CAS, 82 minutes for EM/IM and 79 minutes for IM/IM. 68 patients completed 10-year follow-up. We failed to find significant differences in knee pain $(\mathrm{p}=0.11), \mathrm{knee}$ function $(\mathrm{p}=0.24)$, satisfaction $(\mathrm{p}=0.43)$ or health status physical component score $(\mathrm{p}=0.06)$ or mental component score $(\mathrm{p}=0.29)$ at 10 years.

Conclusion: 10-year results of this study indicate that compared to conventional techniques, CAS fails to provide superior long-term functional outcomes for patients undergoing TKA.

Keywords: arthroplasty, computer-navigation, post-operative outcomes

\section{Introduction}

Traditionally total knee arthroplasty (TKA) has been undertaken using intra- and extramedullary alignment jigs to measure and resect the distal femur and proximal tibia, aiming for a neutral alignment. The implants are seated perpendicular to the mechanical axis of the femur and tibia, with surgeons aiming for the overall mechanical alignment to be neutral $\pm 3^{\circ}{ }^{[1]}$. Conventional thinking suggested deviation $>3^{\circ}$ in either varus or valgus from the neutral axis to be associated with increased rate of implant failure ${ }^{[2-4]}$.

The introduction of computer navigation in arthroplasty aimed to increase precision of implant positioning, lower operative burden, and improve function and survivorship. Computer assisted surgery (CAS) can be broadly classified into image-based and non-image-based navigation systems. Image-based software relies on magnetic resonance imaging (MRI) or computed tomographic (CT) imaging to provide a map of the operative field, which is then used to calculate resection parameters. Imageless navigation relies upon the navigation console registering intraoperative anatomical landmarks to calculate resection. Many trials have validated these technologies, and shown them to be reliable in improving the accuracy of implant position within $\pm 3^{\circ}$ of the limb's mechanical axis ${ }^{[5,6]}$

The implications of CAS on functional outcomes for patients following TKA have been sparsely reported in the short term $(<2$ years), with very few trials looking at long term $(\geq 10$ years) outcomes. The purpose of this study was to investigate whether CAS technology in TKA provided superior long-term functional outcomes for patients. 


\section{Materials and Methods}

This study on patient reported outcome measures is a follow up paper of a previously published patient series ${ }^{[7,8]}$. This randomised trial followed 107 patients who underwent total knee arthroplasty for a minimum of 10 years. All TKAs were performed by a single surgeon, using the same cemented prosthesis design. Patients were recruited from one centre, between March 2006 and May 2009. Trial participants were randomly assigned into one of three study arms: TKA using CAS technique, TKA with intramedullary (IM) instrumentation guides for both femur and tibia (IM/IM), or TKA with intramedullary femur and extramedullary (EM) tibial jigs (EM/IM).

\section{Ethics}

This trial was approved by the Hospital Human Research and Ethics Committee, and was registered with the Australian New Zealand Clinical Trials Registry (ACTRN12609000404224). The design of this trial and its reporting are based on the CONSORT principles ${ }^{[9]}$.

\section{Randomisation}

Prospectively recruited patients were randomised with equal probability into one of the three treatment arms. This process was conducted with a computer-generated list with random permuted blocks of 3. This sequence of randomisation was concealed prior to patient enrolment, and only made available on the morning of surgery.

\section{Blinding}

Enrolled patients were blinded to their specific treatment arm allocation. Investigators involved in the surgical aspect of this trial were privy to patient allocation. Trial investigators involved in post-operative patient data collection were excluded from any surgical involvement, and remained blinded to the allocation of patients.

\section{Interventions}

All enrolled patients underwent a total knee arthroplasty by a single arthroplasty surgeon (RK). All procedures were performed through the standardised midline incision and medial parapatellar approach. The use of an intraoperative pneumatic tourniquet was constant across all three study groups.

The Genesis II cemented prosthesis (Smith and Nephew, Memphis, USA), was implanted in all patients. Instrumented techniques for patients undergoing TKA with conventional means were carried out using the Genesis II Total Knee System, in accordance with the manufacturer's prescribed techniques. For those patients undergoing navigated TKAs, BrainLab Knee Essential software (BrainLab AG, Feldkirchen, Germany) was utilised for alignment of both femora and tibia.

\section{Rehabilitation}

All patients underwent standardised rehabilitation, with no inter-group variability. This was done under the supervision and guidance of physiotherapists. Full weight bearing on the operative limb was permitted from return to ward, and walking aids were used as adjuncts for balance. Passive and active range of motion exercises were commenced on day one post-operatively. In-dwelling urinary catheters were inserted prior to commencement of surgery, and in accordance with our post lower limb arthroplasty protocol, were removed once patients were able to mobilise to the toilet.

\section{Outcomes}

Primary outcomes were post-operative knee function measured using the Oxford Knee Score (OKS) ${ }^{[10]}$. Information pertaining to pain was collected through the use of a Numerical Knee Pain Rating scale ${ }^{[11]}$.

Secondary outcomes included Short Form Health Survey (SF12) ${ }^{[12]}$ assessed health status in physical (PCS) and mental (MCS) components. Patient satisfaction with the knee was measured with the Likert satisfaction survey scale. These questionnaires were sent to patients with a return-paid envelope at timed intervals; pre-operatively, 5- and 10-year post-operatively.

In addition, a thorough screening of hospital patient records was conducted for each patient to identify any medical complications

\section{Statistical Analysis}

This study follows an earlier paper investigating component positioning ${ }^{[7]}$. The 10-year functional data was analysed using descriptive statistics, based on frequencies and means. Univariate analysis between groups include $\chi^{2}$ tests for categorical comparisons, and non-parametric Kruskal-Wallis $\mathrm{H}$ tests for continuous outcomes. Outcome data was also stratified by sex but did not influence the results and therefore the unadjusted data is presented. Data were analysed using IBM SPSS version 20.5 (Armonk, NY). P-values <0.05 were considered statistically significant

\section{Results}

The patients averaged 70 years of age at time of surgery, with 48 males and 59 females. Mean surgical time was collected prospectively in this cohort of patients previously studied by Blakeney et al. ${ }^{[7]} ; 112$ minutes for CAS (SD 25.87). 82 minutes for EM/IM (SD 20.4) and 79 minutes for IM/IM (S 18.69).

68 patients were available for follow-up at 10-years post initial surgery, with comparable rates of loss to follow-up within each group. (Fig1)

Table 1: Patient and surgical demographics

\begin{tabular}{|c|c|c|c|c|}
\hline Mean (Standard Deviation) & CAS n=36 & EM/IM n=35 & IM/IM n=36 & P value \\
\hline Age (years) & $67.8(8.85)$ & $71.2(9.47)$ & $70.2(7.14)$ & 0.263 \\
\hline BMI (kgm $\left.{ }^{-2}\right)$ & $30.1(4)$ & $29.2(5.46)$ & $29.7(7.86)$ & 0.227 \\
\hline Knee Function (OKS) & $19(6.28)$ & $19(7.76)$ & $19(6.62)$ & 0.915 \\
\hline SF-12 PCS & $29.8(6.64)$ & $30.1(5.71)$ & $30.1(8.44)$ & 0.990 \\
\hline SF-12 MCS & $47.1(12.48)$ & $47.1(10.44)$ & $47.2(11.81)$ & 0.999 \\
\hline Ratios & & & & \\
\hline Gender (M:F) & $15: 8$ & $6: 17$ & $5: 17$ & 0.015 \\
\hline Laterality (R:L) & $11: 12$ & $16: 7$ & $12: 10$ & \\
\hline
\end{tabular}




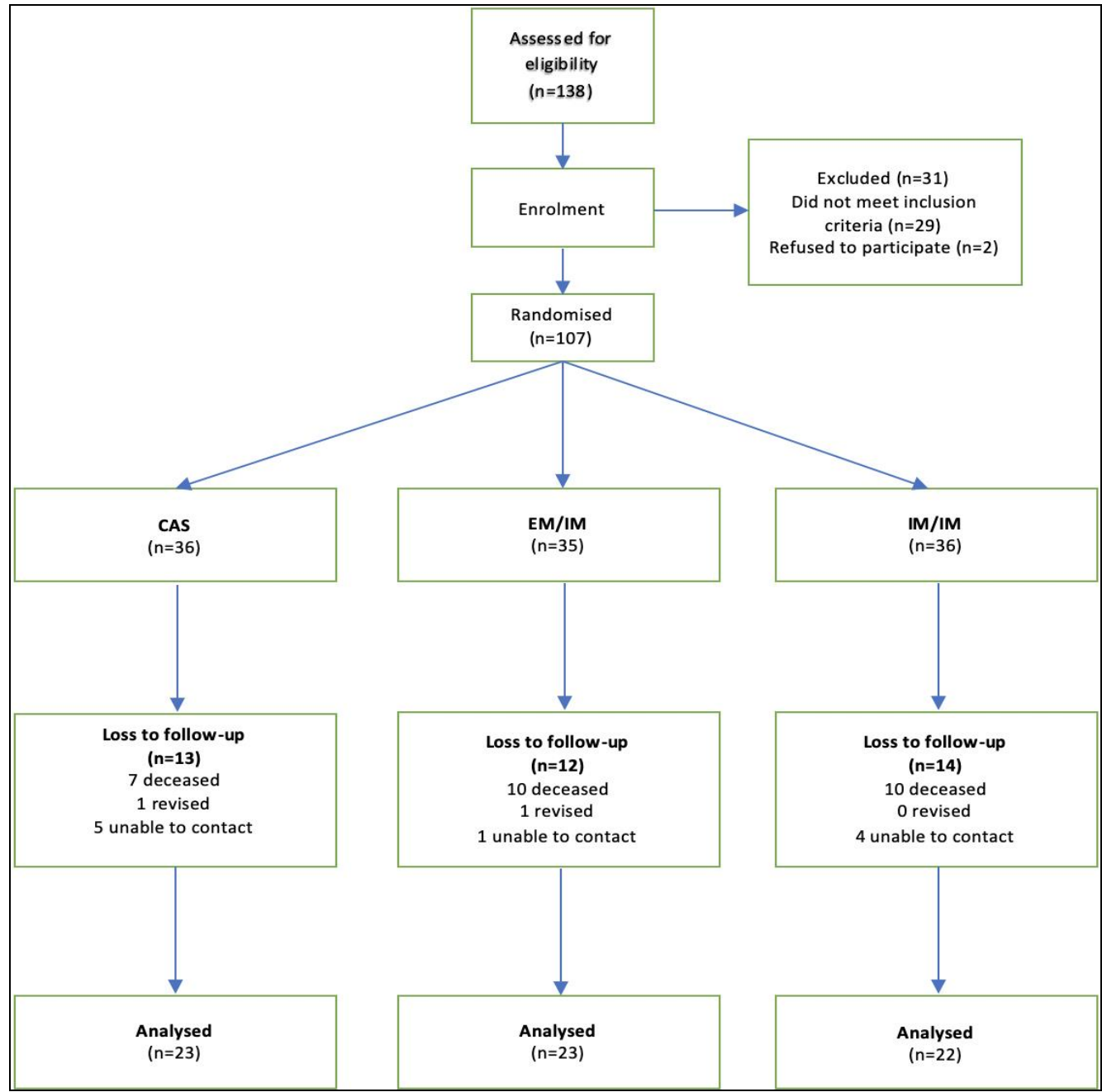

Fig 1: Patient flow

Average age, BMI and knee function at time of surgery were comparable among the 3 study cohorts, detailed in table 1 .
Mean OKS scores demonstrated improvement and attainment of excellent knee function $(>38 / 48)$ by all three TKA implantation techniques at 5 - and 10-years following surgery.

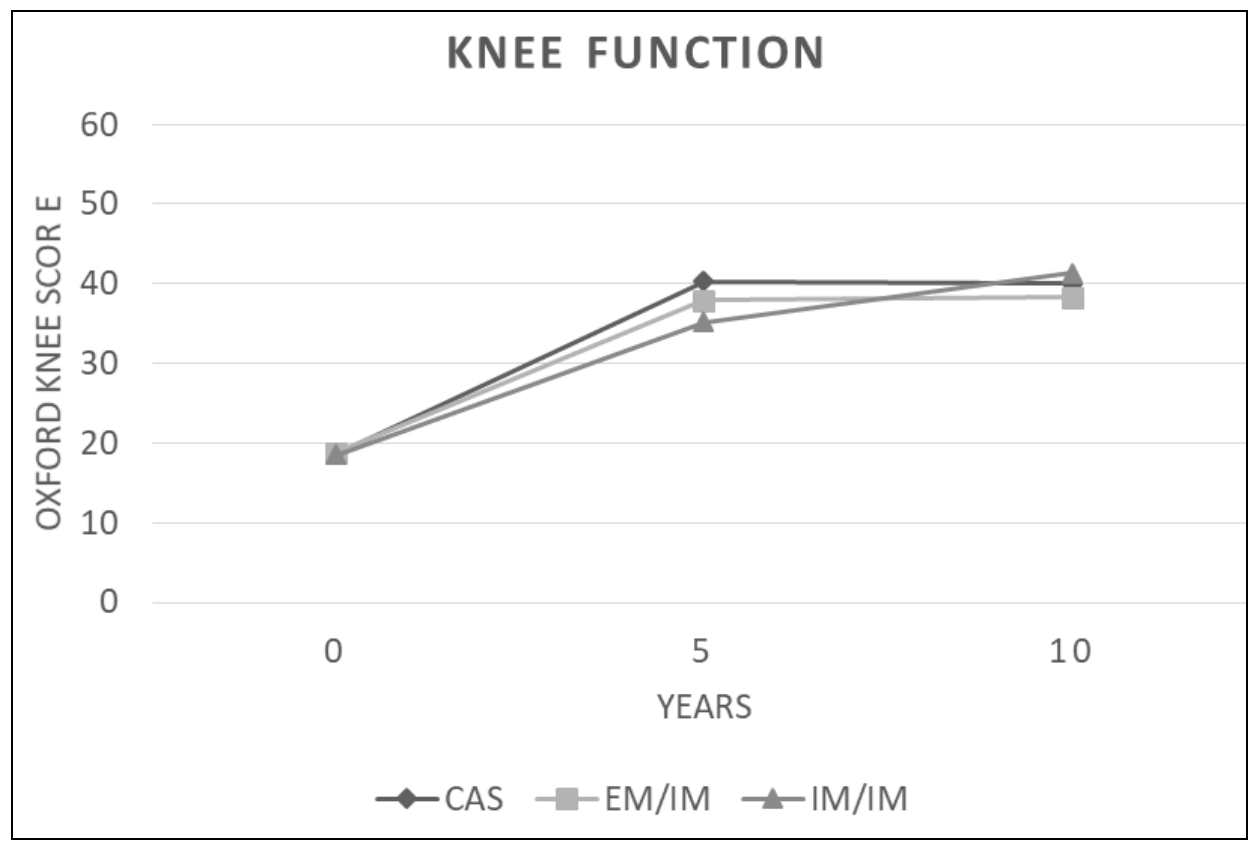

Graph 1: Oxford Knee Scores

We failed to find significant differences in knee pain $(\mathrm{p}=0.11)$, knee function $(\mathrm{p}=0.24)$, satisfaction $(\mathrm{p}=0.43)$ or health status (PCS $\mathrm{p}=0.06$, MCS $\mathrm{p}=0.29$ ) at 10 years. The physical component of the SF12 health score was better, but not significantly so, in the CAS group. Results are detailed in Table 2. 
Table 2: 10-Year Mean Patient Reported Outcome Measures

\begin{tabular}{|c|c|c|c|c|}
\hline Mean (Standard Deviation) & CAS n=23 & EM/IM n=23 & IM/IM n=22 & p-value \\
\hline OKS & $40(10.33)$ & $38(7.95)$ & $41(5.92)$ & 0.24 \\
\hline Pain & $2.4 / 10(2.62)$ & $2.4 / 10(2.35)$ & $1 / 10(1.65)$ & 0.11 \\
\hline Satisfaction & $91 \%$ & $77 \%$ & $78 \%$ & 0.43 \\
\hline SF-12 PCS & $44.7(12.49)$ & $36.8(10.08)$ & $38.8(12.69)$ & 0.06 \\
\hline SF-12 MCS & $50.2(9.43)$ & $51.4(11.15)$ & $54.1(8.89)$ & 0.29 \\
\hline
\end{tabular}

\section{Discussion}

CAS has been shown to provide superior radiological outcomes, when compared to conventional intra- or extramedullary techniques, with higher frequencies of postoperative limb alignment within $+/-3^{\circ}$ of a neutral mechanical axis [7, 13-16]. Advocates of this technology suggest that through better implant alignment, patients will benefit from improved long-term functional outcomes and survivorship of their prosthesis ${ }^{[17]}$. However, these claims have not been substantiated by high-quality randomised trials with long-term follow-up. The purpose of our trial was to determine if the use of CAS technology in total knee arthroplasty provided any long-term enhancements to patient outcomes. This study failed to find any significant difference in 10-year outcomes between CAS and conventional techniques,

The body of literature comparing CAS to conventional instrumentation for total knee arthroplasty is inconsistent and confounding. Some studies present the reader with persuasive evidence that CAS use in TKA is associated with superior knee function and higher quality of life ${ }^{[16]}$. Krackow et al discuss the superior nature of CAS and its benefits for patient outcomes, compared to conventional instrumentation, for TKA. There is, however, no patient follow-up or assessment of patient outcomes within this study.

More recent trials have found no difference to patient outcomes between TKAs with or without CAS ${ }^{[18-22]}$. A prospective trial with 2-year follow-up, by Spencer et al. ${ }^{[18]}$ noted a correlation between CAS and superior post-operative limb alignment, but this did not translate to a difference in clinical outcomes as measured by the Knee Society score, Western Ontario and McMaster Universities osteoarthritis index (WOMAC), Short Form-36 health survey, Oxford knee score and Bartlett Patellar score. Seon et al. ${ }^{[21]}$, in a prospective trial with 2-year follow-up depicted similar findings, with no differences in post-operative functional outcomes of WOMAC and Hospital for Special Surgery knee scores between TKAs performed with or without navigation. Kamat et al. ${ }^{[20]}$ in a retrospective study with 5-year follow-up had a similar conclusion, with no difference in clinical outcome measures between their navigated and standard TKAs. Participant allocation into either treatment arm was dependent upon availability of the navigation system, and therefore, true randomisation was not applied. The standard instrumentation cohort exhibited greater numbers with postoperative limb alignment outside of $+/-3^{\circ}$ to mechanical axis. Trials investigating long term clinical outcomes following TKA with or without CAS are limited. In a prospective study by Kim et al. ${ }^{[23]}$, clinical outcomes were investigated of 520 (1040 knees) patients undergoing bilateral TKA, with navigation used on one knee, whilst the other was replaced using conventional means. The mean post-operative followup was 10.8 years (range 10-12 years). Unlike the present study, Kim's trial involved 2 senior surgeons, utilising 2 separate TKA systems, each with its own design characteristics. This led to their authors having to perform heterogenous bony resections, based on the inbuilt nuances of each implant. Notwithstanding minor variability in study designs, the results mirrored those of this study. No difference in clinical function, as measured post-operatively with KSS and WOMAC, was noted between study cohorts. Knee range of motion was comparable between both groups preoperatively, with no significant benefit being seen postoperatively through use of CAS.

A prospective trial by Ollivier et al. ${ }^{[24]}$ of 10 -year follow-up also had comparable results. This single surgeon, singlecentre trial aimed to answer if the use of CAS demonstrated any clear difference in patient-reported outcome measures at minimum 10-year follow-up. The number of patients available for follow-up at the end were comparable to the current study at 10 -years post TKA. The results indicate no difference between CAS and conventional groups for SF12, Forgotten Joint and Knee Injury, KSS and Osteoarthritis Outcome scores.

There are some limitations to our study. Whilst adding to a small pool of long-term knowledge, the ability to draw strong conclusions on the basis of our results is limited by sample size, and potential for bias due to the drop-out rates inherent in this long-term trial with a frail elderly population examined 10 years following TKA. A minimum 100 patients is recommended when using the OKS for comparative studies [13]. The generalisability of results may be compromised by the use of a single arthroplasty navigation system, and because all procedures were performed by one surgeon.

It is important to note that whilst our findings do not advocate for the use of CAS technology in mainstream total knee arthroplasty, its use in complex primary and revision arthroplasty was not investigated as part of this trial.

\section{Conclusion}

In our randomised 10-year clinical trial, we found no benefit to patient outcomes, through CAS technology compared to conventional methods in TKA. Adding to this, the longer surgical time for a CAS TKA makes this technique less attractive to the impartial surgeon.

\section{Acknowledgments}

The authors would like to gratefully acknowledge Angela Jacques for her statistical analyses and Sir Charles Gairdner Physiotherapy Department for their administrative support.

\section{Conflicts of Interest}

The two senior authors have the following conflicts of interest to declare:

1. Royalties from a company or supplier - Global Orthopaedic Technology

2. Other financial or material support from a company or supplier - Amplitude, Mathys

All other participating authors have no conflicts of interest.

\section{References}

1. Bargren JH, Blaha JD, Freeman MA. Alignment in total knee arthroplasty. Correlated biomechanical and clinical observations. Clin Orthop Relat R 1983, 178-83

2. Bonner TJ, Eardley WGP, Patterson P, Gregg PJ. The 
effect of post-operative mechanical axis alignment on the survival of primary total knee replacements after a follow-up of 15 years. J Bone Jt Surg Br 2011;93:121722. https://doi.org/10.1302/0301-620x.93b9.26573

3. Tew M, Waugh W. Tibiofemoral alignment and the results of knee replacement. J Bone Jt Surg Br Volume 1985;67-B:551-556. https://doi.org/10.1302/0301-620x.67b4.4030849

4. Jeffery R, Morris R, Denham R. Coronal alignment after total knee replacement. J Bone Jt Surg Br 1991;73B:709-714. https://doi.org/10.1302/0301-620x.73b5.1894655

5. Bäthis H, Perlick L, Tingart $\mathrm{M}$ et al. Alignment in total knee arthroplasty A Comparison of Computer-Assisted Surgery With The Conventional Technique. J Bone Jt Surg Br 2004;86-B:682-687. https://doi.org/10.1302/0301-620x.86b5.14927

6. Keyes B, Markel D, Meneghini R. Evaluation of Limb Alignment, Component Positioning, and Function in Primary Total Knee Arthroplasty Using a Pinless Navigation Technique Compared with Conventional Methods. J Knee Surg 2012;26:127-132. https://doi.org/10.1055/s-0032-1319788

7. Blakeney WG, Khan RJK, Wall SJ. Computer-Assisted Techniques Versus Conventional Guides for Component Alignment in Total Knee Arthroplasty. J Bone Jt Surg 2011;93:1377-1384. https://doi.org/10.2106/jbjs.i.01321

8. Blakeney WG, Khan RJK, Palmer JL. Functional outcomes following total knee arthroplasty: A randomised trial comparing computer-assisted surgery with conventional techniques. Knee 2014;21:364-368. https://doi.org/10.1016/j.knee.2013.04.001

9. Moher D, Hopewell S, Schulz KF et al CONSORT 2010 Explanation and Elaboration: updated guidelines for reporting parallel group randomised trials. J Clin Epidemiol 2010;63:e1-e37. https://doi.org/10.1016/j.jclinepi.2010.03.004

10. Murray DW, Fitzpatrick R, Rogers K et al. The use of the Oxford hip and knee scores. J Bone Jt Surg Br 2007;89B:1010-1014. https://doi.org/10.1302/0301-620x.89b8.19424

11. Hawker GA, Mian S, Kendzerska T, French M. Measures of adult pain: Visual Analog Scale for Pain (VAS Pain), Numeric Rating Scale for Pain (NRS Pain), McGill Pain Questionnaire (MPQ), Short-Form McGill Pain Questionnaire (SF-MPQ), Chronic Pain Grade Scale (CPGS), Short Form-36 Bodily Pain Scale (SF-36 BPS), and Measure of Intermittent and Constant Osteoarthritis Pain (ICOAP). Arthrit Care Res 2011;63:S240-S252. https://doi.org/10.1002/acr.20543

12. WARE JE, KOSINSKI M, KELLER SD A 12-Item Short-Form Health Survey. Med Care 1996;34:220-233. https://doi.org/10.1097/00005650-199603000-00003

13. Bauwens K, Matthes G, Wich $\mathrm{M}$ et al. Navigated total knee replacement. A meta-analysis. J Bone Jt Surg Am 2007;89:261-9. https://doi.org/10.2106/00004623-200702000-00005

14. Choong PF, Dowsey MM, Stoney JD. Does Accurate Anatomical Alignment Result in Better Function and Quality of Life? Comparing Conventional and ComputerAssisted Total Knee Arthroplasty. J Arthroplast 2009;24:560-569. https://doi.org/10.1016/j.arth.2008.02.018

15. Dutton AQ, Yeo SJ, Yang KY et al Computer-Assisted Minimally Invasive Total Knee Arthroplasty Compared with Standard Total Knee Arthroplasty. J Bone Jt Surg 2008;90:2-9. https://doi.org/10.2106/jbjs.f.01148

16. Matziolis G, Krocker D, Weiss U, et al A prospective, randomized study of computer-assisted and conventional total knee arthroplasty. Three-dimensional evaluation of implant alignment and rotation. J Bone Jt Surg Am 2007;89:236-43. https://doi.org/10.2106/00004623-200702000-00002

17. Insall JN, Dorr LD, Scott RD, Norman W. Rationale, of The Knee Society Clinical Rating System. Clin Orthop Relat R 1989;248:13-14. https://doi.org/10.1097/00003086-198911000-00004

18. Spencer JM, Chauhan SK, Sloan K et al Computer navigation versus conventional total knee replacement. Bone Joint J 2007;89-B:477-480. https://doi.org/10.1302/0301-620x.89b4.18094

19. Henckel J, Richards R, Lozhkin K et al. Very low-dose computed tomography for planning and outcome measurement in knee replacement. Bone Joint J 2006;88B:1513-1518 https://doi.org/10.1302/0301-620x.88b11.17986

20. Kamat YD, Aurakzai KM, Adhikari AR, et al. Does computer navigation in total knee arthroplasty improve patient outcome at midterm follow-up? Int Orthop 2008;33:1567. https://doi.org/10.1007/s00264-008-06900

21. Seon JK, Park SJ, Lee KB et al. Functional comparison of total knee arthroplasty performed with and without a navigation system. Int Orthop 2009;33:987-990. https://doi.org/10.1007/s00264-008-0594-z

22. Kim YH, Kim JS, Yoon SH. Alignment and orientation of the components in total knee replacement with and without navigation support. Bone Joint J 2007;89-B:471476. https://doi.org/10.1302/0301-620x.89b4.18878

23. Kim YH, Park JW, Kim JS. Computer-Navigated Versus Conventional Total Knee Arthroplasty. J Bone Jt Surg 2012;94:2017-2024. https://doi.org/10.2106/jbjs.1.00142

24. Ollivier M, Parratte S, Lino L, et al. No Benefit of Computer-assisted TKA. Clin Orthop Relat R 2018;476:126-134. https://doi.org/10.1007/s11999.0000000000000021 